OPEN ACCESS

Edited by: Junji Uchino,

Kyoto Prefectural University of Medicine, Japan

Reviewed by:

Brent McHenry,

Bristol Myers Squibb, United States Xabier Mielgo Rubio, Hospital Universitario Fundación Alcorcón, Spain

${ }^{*}$ Correspondence: Hui Guo

guohui@xjtufh.edu.cn

${ }^{+}$These authors have contributed equally to this work and share first authorship

Specialty section: This article was submitted to Thoracic Oncology, a section of the journal Frontiers in Oncology

Received: 20 April 2021 Accepted: 19 August 2021 Published: 13 September 2021

Citation: Jiang $P$, Mao Z, Wang $Q$, Jia $X$, Geng L, Xu H, Jiang L, Yang $C$, Jiao M and Guo H (2021) An Indirect Comparison Between Nivolumab + Ipilimumab + Two Cycles of Chemotherapy vs. Pembrolizumab + Chemotherapy as First-Line Treatment for Metastatic Non-Small Cell Lung Cancer.

Front. Oncol. 11:698199. doi: 10.3389/fonc.2021.698199
An Indirect Comparison Between Nivolumab + Ipilimumab + Two Cycles of Chemotherapy vs. Pembrolizumab + Chemotherapy as First-Line Treatment for Metastatic Non-Small Cell Lung Cancer

\author{
Panpan Jiang ${ }^{1 \dagger}$, Ziyang Mao ${ }^{1 \dagger}$, Qinyang Wang ${ }^{1}$, Xiaohui Jia ${ }^{1}$, Luying Geng ${ }^{1}$, Hong $\mathrm{Xu}^{1}$, \\ Lili Jiang ${ }^{1}$, Chengcheng Yang ${ }^{1}$, Min Jiao ${ }^{1}$ and Hui Guo ${ }^{1,2,3^{*}}$
1 Department of Medical Oncology, The First Affiliated Hospital of Xi'an Jiaotong University, Xi'an, China, ${ }^{2}$ Key Laboratory of Environment and Genes Related to Diseases, Xi'an Jiaotong University, Ministry of Education of China, X'an, China,
${ }^{3}$ Centre for Translational Medicine, The First Affiliated Hospital of Xi'an Jiaotong University, Xi'an, China

Background: Nivolumab + ipilimumab + two cycles chemotherapy ( $\mathrm{N}-\mathrm{I}+$ chemo, intensive immunotherapy but chemo-light) and pembrolizumab + chemotherapy (Pem + chemo) were both recommended as first-line treatment for metastatic non-small cell lung carcinoma (NSCLC) patients. We conducted this indirect comparison to compare the efficacy of and safety between these two treatments for providing reference for decision making.

Methods: Relevant databases were searched for eligible trials. A well-accepted adjusted indirect treatment comparison (ITC) approach was selected to pool efficacy results and safety outcomes. Subgroup analyses were stratified according to PD-L1 expression and clinical characteristics.

Results: Four eligible randomized trials (CheckMate9LA, KEYNOTE-021G, KEYNOTE 189, KEYNOTE 407) involving 2017 patients were available to analyze. The ITC results suggested that $\mathrm{N}-\mathrm{I}+$ chemo is comparable to Pem + chemo in OS $(\mathrm{HR} 1.03,95 \% \mathrm{Cl} 0.82$ 1.30) and ORR (RR 0.81, 95\% Cl 0.62-1.06), but tended to yield inferior PFS (HR 1.28, 95\% Cl 1.04-1.59) than did Pem + chemo. As for safety profiles, N-I + chemo showed no significant difference relative to Pem + chemo in any grade adverse events: (RR 1.03, 95\% $\mathrm{Cl}$ 0.99-1.10), but demonstrated reduced toxicity in chemo-related adverse events, such as anemia (RR 0.63, 95\% Cl 0.49-0.81), neutropenia (RR0.51, 95\% Cl 0.33-0.79), and thrombocytopenia (RR 0.38, 95\% Cl 0.21-0.69). 


\section{Conclusions: $\mathrm{N}-\mathrm{I}+$ chemo is a promising treatment option for providing comparable OS related to Pem + chemo. However, for never smoker female patients, Pem + chemo is preferable to choose for demonstrating favorable OS benefit than $\mathrm{N}-\mathrm{I}+$ chemo.}

Keywords: pembrolizumab, nivolumab, ipilimumab, non-small cell lung cancer, efficacy, safety

\section{INTRODUCTION}

In the last decade, the treatment landscape of lung cancer has been revolutionized to the era of immunotherapy, and the most prominent representatives are immune checkpoint inhibitors (ICIs), including medications targeting programmed death receptor 1 (PD-1), programmed death-ligand 1 (PD-L1), and cytotoxic T-lymphocyte-associated protein 4 (CTLA-4) $(1,2)$. Advances in the $1 \mathrm{~L}$ immunotherapy treatment of driver mutation-negative metastasis non-small cell lung cancer is remarkable (3). Multiple phase III clinical trials have verified the superior efficacy and acceptable toxicity of ICIs in this population, alone or with other regimens (4-7). However, only $20 \%$ of patients obtain long-term survival benefit from a single agent of ICIs (8). Accumulated evidence indicated that a synergistic effect of different regimens contributes to the prolonged survival outcomes $(9,10)$. Therefore, combination therapy was explored to improve the efficacy and expand the beneficiaries. Specifically, chemo-immunotherapy combinations demonstrated particularly encouraging survival outcomes, and among multiple regimens, pembrolizumab + chemotherapy $($ Pem + chemo) seemed to yield a better survival benefit $(11,12)$.

Most recently, a new combination approach, nivolumab and ipilimumab in combination with only two cycles of chemotherapy (N-I + chemo), was designed to administrate in CheckMate9LA (NCT 03,215,706) (13) and showed significantly prolonged OS compared with chemotherapy alone ( $\mathrm{HR}=0.66,95 \% \mathrm{CI}$ : 0.55 to 0.80) after 12.7 months of follow-up. Thus, this chemo-light combination was approved for previously untreated metastatic NSCLC regardless of PD-L1 expression by the United States Food and Drug Administration (FDA) regulatory in May 2020 (14). N-I + chemo is considered as a new promising treatment option which is associated with improved efficacy in the combination of distinct immune checkpoint inhibitors by functioning in complementary mechanisms. Besides, N-I + chemo is well tolerable due to the short cycle chemotherapeutic agents.

$\mathrm{N}-\mathrm{I}+$ chemo and Pem + chemo, representing two different combination strategies, were both recommended as first-line treatment for metastatic NSCLC patients without EGFR/ALK mutation. However, there is no available direct comparisons between these two regimens to provide a reference for decision making. Indirect comparison methods (ITC) is an established approach to compare interventions from different trials and the reliability and validity of results has been confirmed to be highly consistent with direct comparisons $(15,16)$. Thus, we use this method to investigate the potential efficacy and safety difference among N-I + chemo and Pem + chemo in patients with NSCLC in order to offer robust evidence for clinicians, patients, and policy makers to make choices based on comprehensive considerations. Subgroup analysis stratification according to the status of PD-L1 expression and patients' characteristics also be conducted to guide clinic individualized treatment.

\section{METHODS}

\section{Study Eligibility}

We conducted a systematic search on PubMed, Embase, and the Cochrane Central Register of Controlled Trials databases to identify eligible randomized controlled trials performed before January 2021, comparing the efficacy of first-line N-I + chemo or Pem + chemo for metastasis NSCLC patients. Language was restricted to English. Relevant international conferences, such as American Society of Clinical Oncology (ASCO), European Society for Medical Oncology (ESMO), American Association for Cancer Research for Medical Oncology (AACR), and World Conference on Lung Cancer (WCLC) of recent years were also retrieved to avoid missing data. Keywords and relevant variants including "pembrolizumab," "nivolumab," "ipilimumab," "non-small-cell lung cancer," and "randomized controlled trial" were used to build a search strategy. Study screening and evaluation were conducted by two investigators independently, with disagreements solved by discussion.

\section{Data Extraction}

Two investigators (P.P.J. and Z.Y.M.) independently examined eligible studies in detail and extracted relevant data. As for conflicts, a superior investigator is involved to adjudicate. The outcomes of this study in which we were most interested included overall survival (OS), progression-free survival (PFS), and objective response rate (ORR). We also extracted the following data: treatment-related adverse events (TRAEs), events leading to discontinuation of treatment, and events leading to death. The hazard ratios (HRs) and their 95\% confidence intervals (CIs) were acquired for the analysis of survival (OS and PFS), while the dichotomous data was available for ORR and TRAEs analysis. Subgroup analyses were also conducted in OS and PFS according to different PD-L1 expression, histology, sex, age, smoking status and Eastern Cooperative Oncology Group performance status (ECOG PS).

\section{Statistical Analysis}

Traditional meta-analyses were performed to compare the efficacy and safety of Pem + chemo and chemo. The adjusted indirect comparison of N-I + chemo versus Pem + chemo were achieved through an common intervention (chemotherapy), while there is direct comparison between N-I+chemo vs chemo and Pem+chemo vs chemo. The log HR of the indirect comparison was estimated as the following formula: 
$\log \mathrm{HR}_{\mathrm{AB}}=\log \mathrm{HR}_{\mathrm{AC}}-\log \mathrm{HR}_{\mathrm{BC}}$, and its standard error (SE) for the $\log \mathrm{HR}$ was SE $\left(\log \mathrm{HR}_{\mathrm{AB}}\right)=\sqrt{ }\left(\mathrm{SE}\left(\log \mathrm{HR}_{\mathrm{AC}}\right)^{2}+\mathrm{SE}(\log \right.$ $\left.\left.\mathrm{HR}_{\mathrm{AB}}\right)^{2}\right)$. RR was calculated similarly using this manner $(17,18)$. TATA 12.0 (Stata Corporation, College Station, TX) is available for all statistical analyses in this study. A two-sided $\mathrm{P}$ of $<.05$ is statistically significant.

\section{RESULTS}

\section{Characteristics of the Eligible Studies Study Selection and Quality Assessment}

After rigorous selection, 4 relevant RCTs $(5,6,13,19)$ (involving 2017 patients) were identified for inclusion. A specific selection process is illustrated in Figure 1.

Updated outcomes were selected for analysis in our study for concluding reliable results. Among 4 included studies, 3 were related to Pem + chemo, and only 1 trial was on N-I + chemo. All studies but KEYNOTE 021G (NCT 02,039,674) were phase 3, international, multicenter trials. Regarding the risk of bias, the Cochrane Collaboration's risk of bias tool (20) was followed to judge. The main bias was due to insufficient follow-up time and the deficiency of data concerning immune-related adverse events in CheckMate 9LA (NCT 03,215,706). In general, bias assessment results support the high evidence level of our study (Supplemental Table 1). Basic characteristics of included studies are presented in Table 1. As can be seen from the table, the studies are basically comparable in terms of study design and patient population. Clinical outcomes available for each included RCT are summarized in Table 2.

\section{Primary and Exploratory Outcomes}

In direct comparison, Pem + chemo appears superior to chemo both in PFS (HR 0.53, 95\% CI 0.47-0.61) and OS (HR 0.64, 95\% CI 0.56-0.73) (Supplemental Figure 1), while N-I + chemo also showed advantages over chemo in PFS (HR 0.68, 95\% CI 0.57 0.82 ) and OS (HR 0.66, 95\% CI 0.55-0.80) (Figure 2A). Besides, Pem + chemo demonstrated improved ORR compared with chemo (HR1.90, 95\% CI 1.64-2.19). Similar ORR benefit was observed in N-I + chemo vs. chemo (HR 1.54, 95\% CI 1.23-1.92). In indirect comparison, $\mathrm{N}-\mathrm{I}+$ chemo showed no significant difference to Pem + chemo in OS (HR 1.03, 95\% CI 0.82-1.30) but is associated with inferior PFS (HR 1.28, 95\% CI 1.04-1.59). Regarding ORR, N-I + chemo produced comparable benefits over Pem + chemo (RR 0.81, 95\% CI 0.62-1.06) (Figure 2A).

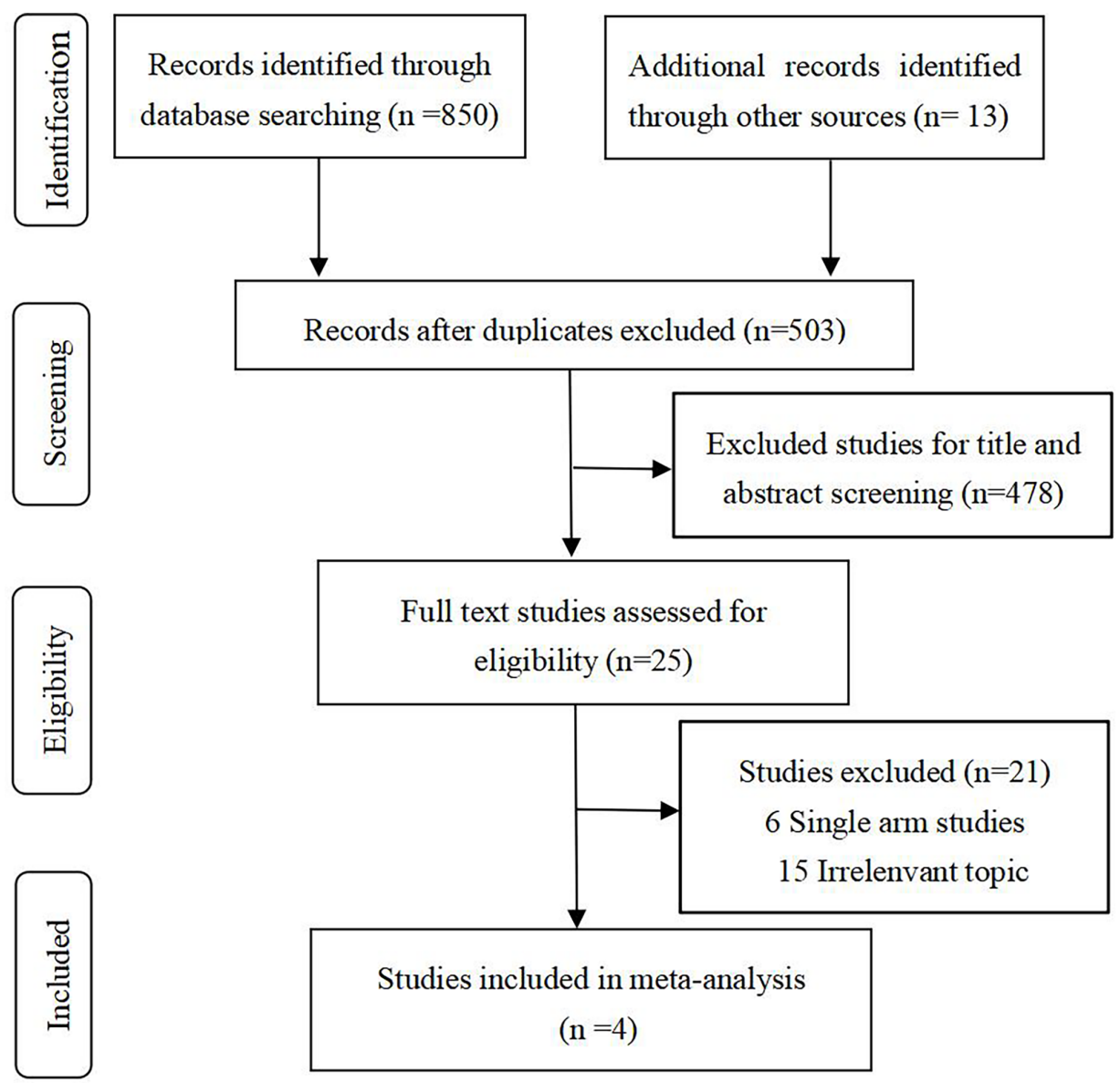

FIGURE 1 | Study flow diagram. 
TABLE 1 | Baseline characteristics of included trials.

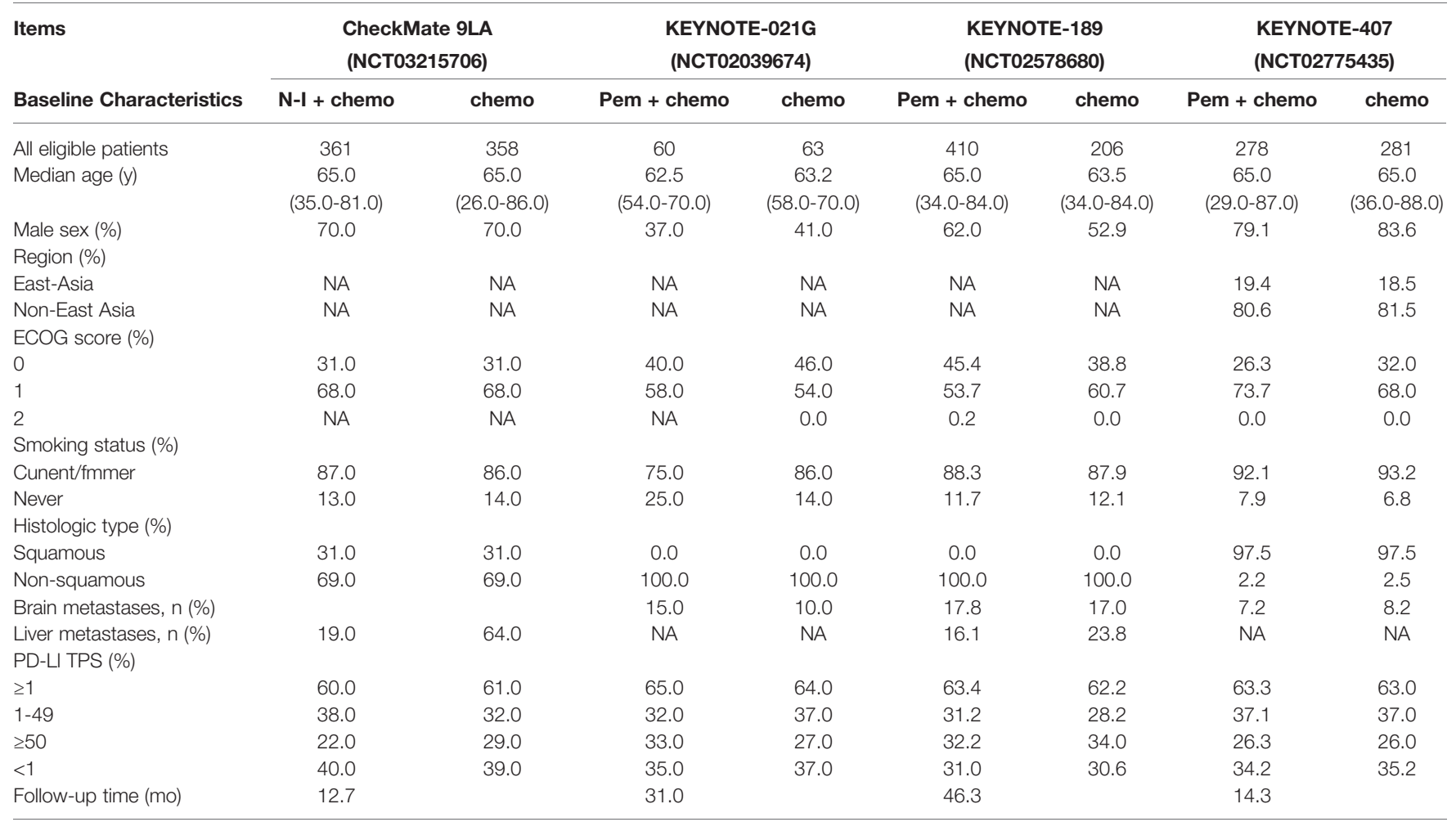

N-1, nivolumab plus ipilimumab; Pem, pembrolizumab; chemo, chemotherapy; $y$, years; NA, not available; ECOG, Eastem Cooperative Oncology Group; PS, perfmmance status; PD-L1 TPS, PD-L1 tumor proportion score; mo, months.

TABLE 2 | Infmmation on prima1y outcome of the studies included in meta-analysis.

\begin{tabular}{|c|c|c|c|c|c|c|c|c|c|c|c|c|c|c|c|c|}
\hline \multirow[t]{3}{*}{ Source } & \multicolumn{5}{|c|}{ HR for OS $(95 \% \mathrm{Cl})$} & \multirow{3}{*}{$\begin{array}{c}\text { HR for PFS } \\
(95 \% \mathrm{Cl})\end{array}$} & \multicolumn{2}{|c|}{ ORR(\%) } & \multicolumn{8}{|c|}{ Incidence of TRAEs (\%) } \\
\hline & \multirow[t]{2}{*}{ Overall } & \multirow[t]{2}{*}{$\begin{array}{l}\mathrm{PD}-\mathrm{LI} \geq \\
50 \%\end{array}$} & \multirow[t]{2}{*}{$\begin{array}{c}1 \% \leq \text { PD-LI } \\
<50 \%\end{array}$} & \multirow[t]{2}{*}{ PD-L1 $\geq \%$} & \multirow[t]{2}{*}{ PD-LI <1\% } & & \multirow[t]{2}{*}{ EM } & \multirow[t]{2}{*}{$\mathrm{CM}$} & \multicolumn{8}{|c|}{$\begin{array}{l}\text { Grade 1-5 AEs Grade 3-5 AEsleading to } \\
\text { discontinuation leading to death }\end{array}$} \\
\hline & & & & & & & & & EM & $\mathrm{CM}$ & EM & CM & EM & $\mathrm{CM}$ & EM & $\mathrm{CM}$ \\
\hline $\begin{array}{l}\text { CheckMate } \\
\text { 9AL }\end{array}$ & $\begin{array}{l}0.66(0.55- \\
0.80)\end{array}$ & $\begin{array}{l}0.66(0.44- \\
0.99)\end{array}$ & $\begin{array}{l}0.61(0.44- \\
0.84)\end{array}$ & $\begin{array}{l}0.64(0.50- \\
0.82)\end{array}$ & $\begin{array}{l}0.62(0.45- \\
0.85)\end{array}$ & $0.68(0.57-0.82)$ & 38.0 & 25.0 & 92.0 & 88.0 & 49.0 & 40.0 & 19.0 & 7.0 & 2.0 & 2.0 \\
\hline Keynote189 & $\begin{array}{l}0.60(0.50- \\
0.72)\end{array}$ & $\begin{array}{l}0.71(0.50- \\
1.00)\end{array}$ & $\begin{array}{l}0.66(0.47- \\
0.93)\end{array}$ & & $\begin{array}{l}0.52(0.37- \\
0.72)\end{array}$ & $0.50(0.41-0.59)$ & 48.3 & 19.9 & 99.8 & 99.0 & 72.1 & 67.3 & 33.6 & 16.3 & 7.2 & 6.9 \\
\hline $\begin{array}{l}\text { KEYNOTE- } \\
407\end{array}$ & $\begin{array}{l}0.72(0.58- \\
0.88)\end{array}$ & $\begin{array}{l}0.79(0.52- \\
1.21)\end{array}$ & $\begin{array}{l}0.59(0.42- \\
0.84)\end{array}$ & $\begin{array}{l}0.67(0.51- \\
0.87)\end{array}$ & $\begin{array}{l}0.79(0.56- \\
1.11)\end{array}$ & $0.57(0.47-0.69)$ & 62.6 & 38.4 & 98.2 & 97.9 & 69.8 & 68.2 & 27.3 & 13.2 & 4.3 & 1.8 \\
\hline
\end{tabular}

OS, overall survival; PFS, progression-fi.ee survival; ORR, objective response rate; TRAES, treatment-related adverse events; AEs, adverse events; PD-LI, programmed cell death-ligand 1; 95\% Cl, 95\% confidence interval (Cl); EM, experimental a1m; CM, control aim.

\section{Subgroup Analysis According to PD-L1 Expression}

In the PD-L1 TPS $<1 \%$ population, direct comparison revealed improved OS and PFS whether N-I + chemo vs. chemo or Pem + chemo v.s chemo (Supplemental Table 2). Indirect results indicated that OS (HR 0.97, 95\% CI 0.65-1.45) and PFS (HR 1.11, 95\% CI 0.77-1.61) were comparable between N-I + chemo and Pem + chemo in this population (Figure 3).

In the PD-L1TPS $\geq 1 \%$ population, favorable OS and PFS benefit were observed in both the N-I + chemo and the Pem + chemo groups compared to chemo. Indirect comparison showed $\mathrm{N}-\mathrm{I}+$ chemo was not inferior to Pem + chemo in OS (HR 0.95, 95\% CI 0.66-1.39), but showed inferiority in PFS (HR 1.46, 95\% 


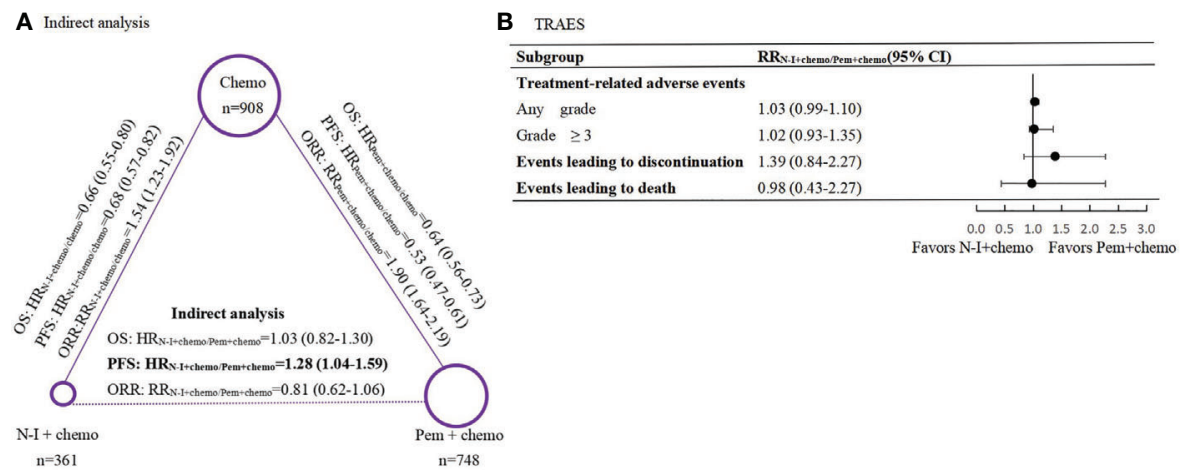

FIGURE 2 | Indirect comparisons of efficacy and safety between N-I + chemo versus Pem + chemo in first-line treatment for patients with advanced NSCLC. (A), Results of indirect analysis for overall survival (OS), progression-free survival (PFS) and objective response rate (ORR) between $\mathrm{N}-\mathrm{I}+\mathrm{chem}$ and Pem + chemo. Each circular represents a treatment. The circle size is associated with the number of enrolled patients. The solid lines represent direct comparisons between the treatments, whereas the dashed line represents the indirect comparison between $\mathrm{N}-\mathrm{I}+$ chemo versus Pem + chemo. (B), Forest plot of risk ratios (RRs) for treatment-related adverse events (TRAEs) between $\mathrm{N}-\mathrm{I}+$ chemo and Pem+chemo. N-I, nivolumab plus ipili.mumab; Pem, pembrolizumab; chemo, chemotherapy.

CI 1.11-1.92). Further subgroup analysis was conducted in patients with PD-L1 TPS $1-49 \%$ and $50 \%$. There was no statistical difference between N-I + chemo and Pem + chemo in terms of OS in these two populations, which were generally consistent with results in the overall $\mathrm{PD}-\mathrm{L} 1 \mathrm{TPS} \geq 1 \%$ population, indicating the convincing nature of the results. With regard to PFS, N-I + chemo appeared inferior to the PFS benefit compared to Pem + chemo in PD-L1 TPS $\geq 50 \%$ population, with $\mathrm{HR}$ of 1.69 (95\% CI 1.08-2.66).

\section{Other Subgroup Analysis}

Due to the inconsistency of stratification criteria in subgroup analysis among different trials, patients $\geqq 65$ years were unavailable for analysis.

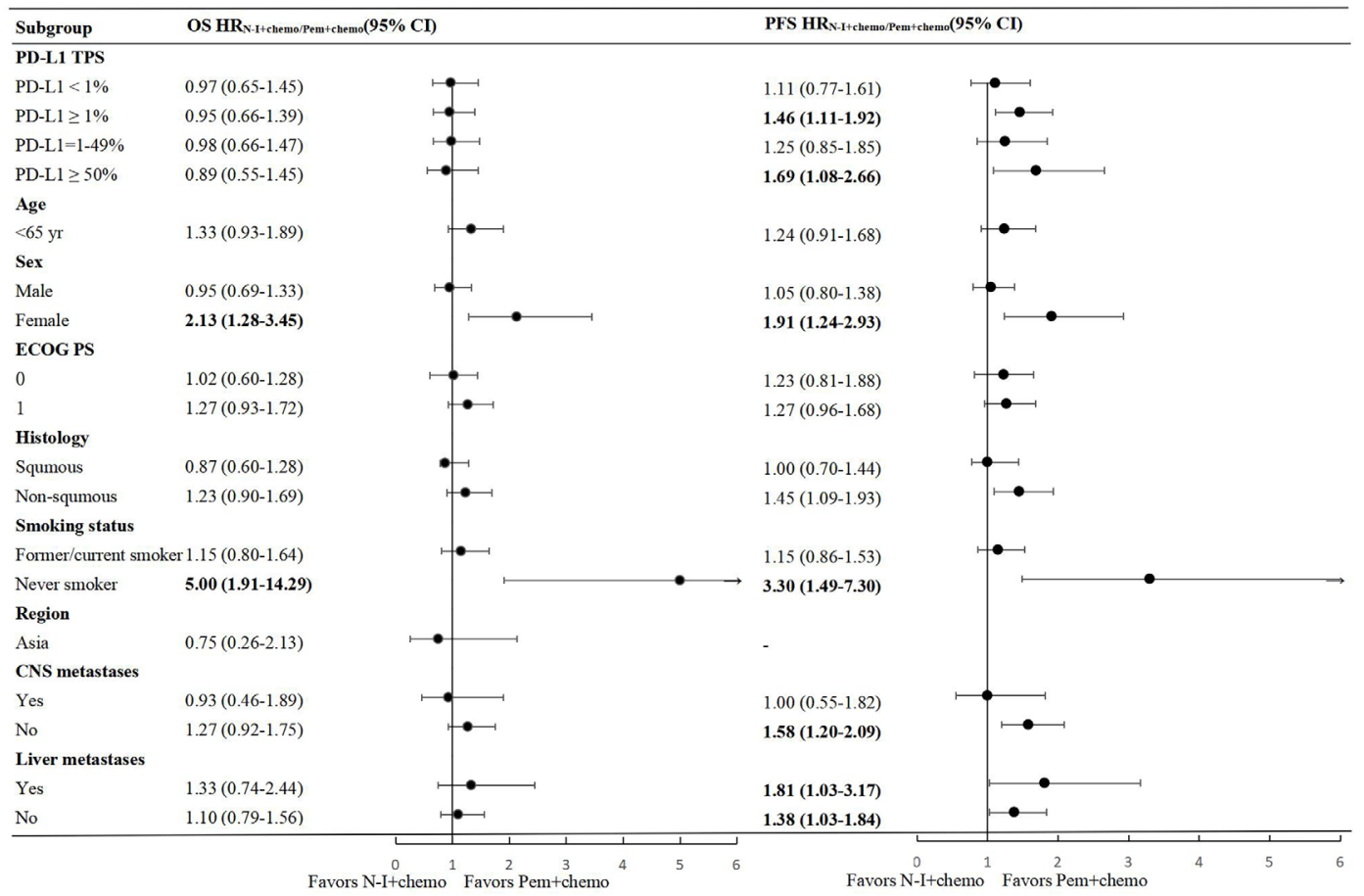

FIGURE 3 | Forest plots of hazard ratios for overall survival and progression free survival in subgroups between N-I + chemo and Pem + chemo. N-I, nivolumab plus ipilimumab; Pem, pembrolizumab; chemo, chemotherapy; Cl, confidence interval; ECOG, Eastern Cooperative Oncology Group; PS, performance status. 
According to indirect comparison, comparable OS and PFS was observed between N-I + chemo and Pem + chemo in pre-stratified subgroups including ECOG PS, CNS metastasis, and liver metastases (Figure 3), which is in accordance with the whole population. Significantly, we found females extended the survival time and postponed the tumor progression from Pem + chemo compared to N-I + chemo, with HR 0.81 (95\% CI 0.29-0.78) and 0.52 (95\% CI 0.34-0.81) for OS and PFS respectively. The same result appears to never smokers, with improved OS (HR 0.20, 95\% CI 0.07-0.52) and PFS (HR 0.30, 95\% CI 0.14-0.67) benefit from Pem + chemo than N-I + chemo. Besides, non-squamous NSCLC patients showed significant advantage from Pem + chemo than N-I + chemo in PFS (HR 0.69, 95\% CI 0.52-0.92) but this advantage was not apparent in OS (HR 0.81, 95\% CI 0.59-1.11).

\section{Safety Analysis}

As for safety profiles, our results demonstrated similar risks across multiple safety endpoints between N-I + chemo and Pem + chemo, including any grade AEs (RR1.03, 95\% CI 0.99-1.10), grade 3-5 AEs (RR 1.02, 95\% CI 0.93-1.35), events leading to drug discontinuation (RR 1.39, 95\% CI 0.84-2.27), and events leading to death (RR 0.98, 95\% CI 0.43-2.27) (Figure 2B). In terms of specific commonly reported TRAEs, N-I + chemo is associated with less hematological toxicity in contrast to Pem + chemo, such as anemia (RR 0.63, 95\% CI 0.49-0.81), neutropenia (RR0.51, 95\% CI 0.33-0.79), and thrombocytopenia (RR 0.38, 95\% CI 0.21-0.69). Nevertheless, the rate of nausea (RR 0.70, 95\% CI 0.55-0.90) and colitis (RR $0.38,95 \%$ CI $0.21-0.69$ ) also occurred less frequently in patients who received N-I + chemo (Table 3). Direct comparisons of safety between Pem + chemo and chemo are presented in Supplemental Figure 2.

\section{DISCUSSION}

$\mathrm{N}-\mathrm{I}+$ chemo and Pem + chemo, representing two different treatment models, were both recommended as first-line treatment options for metastasis NSCLC. It is essential to understand the potential efficacy and safety difference among N-I + chemo and Pem + chemo to provide reference for clinical therapeutic determination. Through comprehensive analysis, our study revealed N-I + chemo (chemo-light) has a comparable OS benefit relative to Pem + chemo, but is associated with a less favorable PFS benefit. Furthermore, any grade AEs as the primary safety endpoint were not observed to be significantly different among N-I + chemo and Pem + chemo. Notably, patients who received N-I + two cycles of chemotherapy experienced less hematologic toxicity.

Current evidence emphasizes the superior survival benefit of Pem + chemo among multiple existing immunotherapies $(11,12,21)$

TABLE 3 | Relative risks for common treatment-related adverse events with $\mathrm{N}-1$ + chemo versus Pem + chemo.

\begin{tabular}{|c|c|c|c|c|}
\hline Treatment-related adverse events & & $\mathrm{RR}_{\mathrm{N}-\mathrm{I}+\mathrm{chemo} / \text { chemo }}(95 \% \mathrm{Cl})$ & $\mathrm{RR}_{\mathrm{Pem}+\mathrm{chemo} / \text { chemo }}(95 \% \mathrm{CJ})$ & $\mathrm{RR}_{\mathrm{N}-\mathrm{J}+\text { chemo/Pem+chemo }}(95 \% \mathrm{CJ})$ \\
\hline \multirow[t]{2}{*}{ Rash } & Any grade & $5.94(3.19-11.04)$ & $1.68(1.28-2.19)$ & 3.57 (1.79-7.14) \\
\hline & Grade $\geq 3$ & $12.67(0.72-224.13)$ & 1.89 (0.63-5.64) & 6.67 (0.31-140.78) \\
\hline \multirow[t]{2}{*}{ Diarrhea } & Any grade & $1.76(1.24-2.50)$ & 1.39 (1.14-1.68) & $1.27(0.85-1.89)$ \\
\hline & Grade $\geq 3$ & $7.31(1.68-31.74)$ & $1.62(0.87-3.01)$ & 4.54 ( 0.92-20) \\
\hline \multirow[t]{2}{*}{ Pruritus } & Any grade & $1.18(0.87-1.60)$ & $2.08(1.36-3.18)$ & $0.57(0.34-0.95)$ \\
\hline & Grade $\geq 3$ & $6.82(0.35-131.63)$ & $0.34(0.01-8.21)$ & 2.00 (0.03-210.04) \\
\hline \multirow[t]{2}{*}{ Fatigue } & Any grade & $1.56(1.07-2.28)$ & 1.09 (0.94-1.270 & $1.43(0.95-2.13)$ \\
\hline & Grade $\geq 3$ & 3.90 (0.83-18.23) & $1.58(0.92-2.70)$ & $2.44(0.48-12.50)$ \\
\hline \multirow[t]{2}{*}{ Decreased appetite } & Any grade & $1.05(0.75-1.46)$ & $0.95(0.79-1.13)$ & $1.11(0.75-1.61)$ \\
\hline & Grade $\geq 3$ & $0.97(0.25-3.87)$ & $1.08(0.38-3.10)$ & $0.90(0.16-5)$ \\
\hline \multirow[t]{2}{*}{ Asthenia } & Any grade & $1.18(0.87-1.60)$ & $0.95(0.76-1.17)$ & $1.23(0.85-1.82)$ \\
\hline & Grade $\geq 3$ & $0.27(0.10-1.37)$ & $1.13(0.62-2.03)$ & 0.33 (0.09-1.22) \\
\hline \multirow[t]{2}{*}{ Nausea } & Any grade & $0.75(0.60-0.93)$ & $1.07(0.95-1.21)$ & $0.70(0.55-0.90)$ \\
\hline & Grade $\geq 3$ & $1.62(0.39-6.75)$ & $1.05(0.52-2.14)$ & 1.54 (0.32-7.69) \\
\hline \multirow[t]{2}{*}{ Vomiting } & Any grade & $0.90(0.63-1.29)$ & 1.30 (1.04-1.63) & $0.69(0.45-1.06)$ \\
\hline & Grade $\geq 3$ & $1.17(0.36-3.80)$ & $0.91(0.43-1.93)$ & $1.28(0.32-5.26)$ \\
\hline \multirow[t]{2}{*}{ Constipation } & Any grade & $0.78(0.50-1.21)$ & $1.13(0.94-1.36)$ & $0.69(0.43-1.11)$ \\
\hline & Grade $\geq 3$ & & $1.08(0.28-4.11)$ & \\
\hline \multirow[t]{2}{*}{ Anemia } & Any grade & $0.61(0.49-0.77)$ & $0.97(0.87-1.09)$ & $0.63(0.49-0.81)$ \\
\hline & Grade $\geq 3$ & $0.40(0.25-0.65)$ & $0.93(0.73-1.20)$ & $0.43(0.25-0.74)$ \\
\hline \multirow[t]{2}{*}{ Neutrophil count decreased } & Any grade & $0.27(0.63-2.59)$ & $1.38(0.58-3.26)$ & $0.92(0.30-2.78)$ \\
\hline & Grade $\geq 3$ & $2.20(0.42-11.59)$ & 1.05 (0.82-1.34) & 0.79 (0.39-1.59) \\
\hline \multirow[t]{2}{*}{ Neutropenia } & Any grade & $0.58(0.39-0.86)$ & $1.13(0.95-1.35)$ & $0.51(033-0.79)$ \\
\hline & Grade $\geq 3$ & $0.71(0.43-1.17)$ & $1.05(0.82-1.34)$ & $0.68(0.39-1.19)$ \\
\hline \multirow[t]{2}{*}{ Thrombocytopenia } & Any grade & $0.49(0.28-0.86)$ & 1.30 (1.04-1.63) & $0.38(0.21-0.69)$ \\
\hline & Grade $\geq 3$ & $1.19(0.50-2.84)$ & $1.22(0.80-1.85)$ & $0.97(0.22-4.35)$ \\
\hline \multirow[t]{2}{*}{ Colitis } & Any grade & $10.72(1.39-82.62)$ & $3.69(1.33-10.23)$ & $0.38(0.21-0.69)$ \\
\hline & Grade $\geq 3$ & $10.72(0.60-193.21)$ & $3.11(0.92-10.51)$ & $3.45(0.15-100)$ \\
\hline \multirow[t]{2}{*}{ Hypothyroidism } & Any grade & 55.57 (7.74-399.08) & $3.88(1.83-8.25)$ & $14.29(1.73-117.83)$ \\
\hline & Grade $\geq 3$ & $2.92(0.01-71.55)$ & 3.58 (0.43-29.63) & $0.81(0.02-33.33)$ \\
\hline \multirow[t]{2}{*}{ Adrenal insufficency } & Any grade & $24.37(1.45-410.08)$ & $1.73(0.29-10.20)$ & $14.29(0.50-403.00)$ \\
\hline & Grade $\geq 3$ & 8.77 (0.47-162.36) & $1.18(0.18-7.97)$ & 7.69 (0.24-249.20) \\
\hline
\end{tabular}

$N$-I, nivolumab plus ipilimumab; Pem, pembrolizumab; chemo, chemotherapy; $R R$, relative risk. 
in first-line treatment for metastasis NSCLC. Our results showed $\mathrm{N}-\mathrm{I}+$ chemo as a chemo-light therapy has comparable OS benefit compared with Pem + chemo, which encourages patients to choose $\mathrm{N}-\mathrm{I}+$ chemo under similar OS benefit while chemotherapy is intolerable. Considerable OS benefit of this chemo-light combination therapy is attributed to several aspects. First, short cycle chemotherapy can increase tumor immunogenicity by eliminating tumor cells and releasing antigen $(10,22)$, and is also associated with enhanced PD-L1 expression and potentiated T cellmediated cytotoxicity while treated with nivolumab (23). Moreover, distinct immune checkpoint inhibitors function in complementary mechanisms with improved efficacy $(24,25)$. As for insufficient PFS benefit of N-I + chemo compared to Pem + chemo revealed in our study, possible explanation may include inadequate follow-up time and unsatisfactory synergy of nivolumab and ipilimumab. Mature data updated in the future will be discussed further.

Given the expression status of PD-L1 is established biomarkers of the efficacy of immunotherapy (26), subgroup analyses according to different level of PD-L1 were conducted to guide more individualized treatment. However, no significant OS benefit difference was observed across different PD-L1 levels, which is generally identical with the results in the whole population.

Unexpectedly, our study suggests Pem + chemo appears to have significantly superior efficacy in deferring tumor progression compared with $\mathrm{N}-\mathrm{I}+$ chemo in patients with PD-L1 TPS $\geq 50 \%$. This finding overturns our previous hypothesis that patients with high PD-L1 expression can benefit more from dual immune inhibitors. Similarly, in KEYNOTE-598 (NCT 03,302,234) (27), Pem + ipilimumab failed to improve efficacy compared to pembrolizumab monotherapy in the first-line treatment of metastatic NSCLC patients with PD-L1 > 50\%, which also suggests the predictive value of $\mathrm{PD}-\mathrm{L} 1$ expression is unavailable in dual immune inhibitors. Thus, valuable predictive biomarkers for $\mathrm{N}-\mathrm{I}+$ chemo need further investigation to identify potential beneficiaries. With regard to the other subgroup analyses, comparable OS and PFS benefits were observed between N-I + chemo and Pem + chemo in most groups, but this result is missing females and never smokers. Intriguingly, Pem + chemo appeared more effective than N-I + chemo in females and never smokers, and there are multiple overlaps in these two populations. This result is in conformity to current research evidence. First, studies have reported that female tumors tend to have less cancer-associated antigens than male tumors $(28,29)$. This indicates that females have less antigenicity which resulted in a less favorable immunotherapy efficacy in female patients (30). Besides this, the disadvantage in drug pharmacokinetics and pharmacodynamics $(31,32)$ are both considered to be correlated with a compromised efficacy in females. As for efficacy difference observed in smokers and never smokers, potential explanation may be that smokers have different features of gene mutation (33-35) and functions of immunoregulation $(36,37)$, which is conductive to the response of immunotherapy. Most recent evidence further indicated smoking can promote PD-L1 expression (38) and increase TMB (8). All these factors lead to the conclusion that smokers derive more from intensive immunotherapy than never smokers. As for superior survival benefit observed in nonsquamous NSCLC patients who received Pem + chemo treatment, more clinical data are demanded to confirm our findings.
Besides efficacy, the safety profiles are also an essential concern when administrating regiments. Generally, no significant difference was observed in any grade adverse events among N-I + chemo and Pem + chemo in our study. As we expected, short cycle chemotherapy under the model of N-I + chemo was associated with less hematological toxicity, indicating the application superiority for patients unable to suffer long-term standard chemotherapy. With the recent and continuous application of ICIs, increasing attention has been paid to immune-related toxicities. Noteworthy, combined immune blockage with nivolumab and ipilimumab may increase immune-related adverse events $(39,40)$. However, due to the sparse data, thorough immunerelated adverse events are not available to analyze in our study.

As far as we are aware, our study is the first to explore the difference between N-I + chemo and Pem + chemo in NSCLC to provide valuable insight for informing clinical decision making, although of course, more evidence from real-world and direct comparisons is required to support our findings. Another strength of our study was we performed a comprehensive subgroup analysis to explore the potential efficacy difference in patients with different clinical characteristics. Inevitably, several limitations were encountered in our study. First, head-to-head comparison is lacking and there is methodological limitation of indirect comparison for integrating results of trials with heterogeneity. Besides, the immature OS data of N-I + chemo resulting from insufficient follow- up time might lead to a potential bias. Given these limitations, more reliable results based on mature and individual patient's data are required. Additionally, the regimens included in our study represent different combination strategies. However, owing to limited trials included, which treatment strategy is preferable to choose in clinical practice has not been answered. With increasing studies attempting dual checkpoint inhibition combination, future studies evaluating these two treatment models are needed to guide study design and treatment selection.

In conclusion, $\mathrm{N}-\mathrm{I}+$ chemo is a promising treatment option, especially available to patients who are elderly, weak, or unable to suffer through long-term chemotherapy. However, for never smoker female patients, Pem + chemo is preferable to choose for providing superior OS benefit compared to N-I + chemo. Collectively, efficacy and toxicities should be comprehensively taken into consideration and be balanced, for further formulating individualized treatment.

\section{DATA AVAILABILITY STATEMENT}

The original contributions presented in the study are included in the article/Supplementary Material. Further inquiries can be directed to the corresponding author.

\section{AUTHOR CONTRIBUTIONS}

All authors contributed to the article and approved the submitted version. PJ and ZM: Writing - original draft, writing - review and editing. QW and XJ: conceptualization, methodology. LG and HX: investigation, resources, data curation, extracted data from studies, and matched inclusion and exclusion criteria. LJ and CY: software, formal analysis. MJ: visualization, project 
administration. HG: supervision. All authors had full access to all data, critically revised the paper, approved the final analysis, and took responsibility for all aspects of the work to ensure that issues relating to the accuracy or integrity of any part of the work could be appropriately investigated and resolved.

\section{FUNDING}

This work was supported by Targeted Therapy Fund of Chinese Society of Clinical Oncology (Y-HR2015-139) and Chinese Society of Clinical Oncology MSD Cancer Research (Y-MSD2020-0247).

\section{REFERENCES}

1. Hanna NH, Robinson AG, Temin S, Baker S Jr, Brahmer JR, Ellis PM, et al. Therapy for Stage IV Non-Small-Cell Lung Cancer With Driver Alterations: ASCO and OH (CCO) Joint Guideline Update. J Clin Oncol (2021) 39(9):104091. doi: $10.1200 /$ JCO.20.03570

2. Goetze TO. Immunotherapy: A New Era in Small-Cell Lung Cancer. Lancet (2019) 394(10212):1884-5. doi: 10.1016/S0140-6736(19)32235-4

3. Ackermann CJ, Adderley H, Ortega-Franco A, Khan A, Reck M, Califano R. First-Line Immune Checkpoint Inhibition for Advanced Non-Small-Cell Lung Cancer: State of the Art and Future Directions. Drugs (2020) 80 (17):1783-97. doi: 10.1007/s40265-020-01409-6

4. Reck M, Rodríguez-Abreu D, Robinson AG, Hui R, Csõszi T, Fülöp A, et al. Pembrolizumab Versus Chemotherapy for PD-L1-Positive Non-Small-Cell Lung Cancer. N Engl J Med (2016) 375(19):1823-33. doi: 10.1056/ NEJMoa1606774

5. Awad MM, Gadgeel SM, Borghaei H, Patnaik A, Yang JC, Powell SF, et al. Long-Term Overall Survival From KEYNOTE-021 Cohort G: Pemetrexed and Carboplatin With or Without Pembrolizumab as First-Line Therapy for Advanced Nonsquamous NSCLC. J Thorac Oncol (2021) 16(1):162-8. doi: $10.1016 /$ j.jtho.2020.09.015

6. Gadgeel S, Rodríguez-Abreu D, Speranza G, Esteban E, Felip E, Dómine M, et al. Updated Analysis From KEYNOTE-189: Pembrolizumab or Placebo Plus Pemetrexed and Platinum for Previously Untreated Metastatic Nonsquamous Non-Small-Cell Lung Cancer. J Clin Oncol (2020) 38 (14):1505-17. doi: 10.1200/JCO.19.03136

7. Hellmann MD, Paz-Ares L, Bernabe Caro R, Zurawski B, Kim SW, Carcereny Costa E, et al. Nivolumab Plus Ipilimumab in Advanced Non-Small-Cell Lung Cancer. N Engl J Med (2019) 381(21):2020-31. doi: 10.1056/NEJMoa1910231

8. Rizvi NA, Hellmann MD, Snyder A, Kvistborg P, Makarov V, Havel JJ, et al. Cancer Immunology. Mutational Landscape Determines Sensitivity to PD-1 Blockade in Non-Small Cell Lung Cancer. Science (2015) 348(6230):124-8. doi: $10.1126 /$ science.aaa1348

9. Manegold C, Dingemans AC, Gray JE, Nakagawa K, Nicolson M, Peters S, et al. The Potential of Combined Immunotherapy and Antiangiogenesis for the Synergistic Treatment of Advanced NSCLC. J Thorac Oncol (2017) 12 (2):194-207. doi: 10.1016/j.jtho.2016.10.003

10. Emens LA, Middleton G. The Interplay of Immunotherapy and Chemotherapy: Harnessing Potential Synergies. Cancer Immunol Res (2015) 3(5):436-43. doi: 10.1158/2326-6066.CIR-15-0064

11. Wang C, Qiao W, Jiang Y, Zhu M, Shao J, Wang T, et al. The Landscape of Immune Checkpoint Inhibitor Plus Chemotherapy Versus Immunotherapy for Advanced non-Small-Cell Lung Cancer: A Systematic Review and MetaAnalysis. J Cell Physiol (2020) 235(5):4913-27. doi: 10.1002/jcp.29371

12. Ando K, Kishino Y, Homma T, Kusumoto S, Yamaoka T, Tanaka A, et al. Nivolumab Plus Ipilimumab Versus Existing Immunotherapies in Patients With PD-L1-Positive Advanced Non-Small Cell Lung Cancer: A Systematic Review and Network Meta-Analysis. Cancers (Basel) (2020) 12(7):1905. doi: 10.3390/cancers12071905

13. Paz-Ares L, Ciuleanu TE, Cobo M, Schenker M, Zurawski B, Menezes J, et al. First-Line Nivolumab Plus Ipilimumab Combined With Two Cycles of

\section{ACKNOWLEDGMENTS}

We are grateful to Dr. Ke-jun $\mathrm{Nan}$ of $\mathrm{Xi}$ 'an International Medical Center Hospital for his valuable guidance on manuscript revision.

\section{SUPPLEMENTARY MATERIAL}

The Supplementary Material for this article can be found online at: https://www.frontiersin.org/articles/10.3389/fonc.2021.698199/ full\#supplementary-material

Chemotherapy in Patients With Non-Small-Cell Lung Cancer (CheckMate 9LA): An International, Randomised, Open-Label, Phase 3 Trial. Lancet Oncol (2021) 22(2):198-211. doi: 10.1016/S1470-2045(20)30641-0

14. Ettinger DS, Wood DE, Aisner DL, Akerley W, Bauman JR, Bharat A, et al. NCCN Guidelines Insights: Non-Small Cell Lung Cancer, Version 2.2021. J Natl Compr Canc Netw (2021) 19(3):254-66. doi: 10.6004/jnccn.2021.0013

15. Song F, Altman DG, Glenny AM, Deeks JJ. Validity of Indirect Comparison for Estimating Efficacy of Competing Interventions: Empirical Evidence From Published Meta-Analyses. BMJ (2003) 326(7387):472. doi: 10.1136/ bmj.326.7387.472

16. Glenny AM, Altman DG, Song F, Sakarovitch C, Deeks JJ, D'Amico R, et al. Indirect Comparisons of Competing Interventions. Health Technol Assess (2005) 9(26):1-iv. doi: 10.3310/hta9260

17. Mauger D, Apter AJ. Indirect Treatment Comparisons and Biologics. J Allergy Clin Immunol Pract (2019) 7(1):131-3. doi: 10.1016/j.jaip.2018.11.008

18. Yang Q, Wei Y, Chen YX, Zhou SW, Jiang ZM, Xie DR. Indirect Comparison Showed Survival Benefit From Adjuvant Chemoradiotherapy in Completely Resected Gastric Cancer With D2 Lymphadenectomy. Gastroenterol Res Pract (2013) 2013:634929. doi: 10.1155/2013/634929

19. Paz-Ares L, Vicente D, Tafreshi A, Robinson A, Soto Parra H, Mazières J, et al. A Randomized, Placebo-Controlled Trial of Pembrolizumab Plus Chemotherapy in Patients With Metastatic Squamous NSCLC: ProtocolSpecified Final Analysis of KEYNOTE-407. J Thorac Oncol (2020) 15 (10):1657-69. doi: 10.1016/j.jtho.2020.06.015

20. Higgins JP, Altman DG, Gøtzsche PC, Jüni P, Moher D, Oxman AD, et al. The Cochrane Collaboration's Tool for Assessing Risk of Bias in Randomised Trials. BMJ (2011) 343:d5928. doi: 10.1136/bmj.d5928

21. Halmos B, Burke T, Kalyvas C, Insinga R, Vandormael K, Frederickson A, et al. A Matching-Adjusted Indirect Comparison of Pembrolizumab + Chemotherapy vs. Nivolumab + Ipilimumab as First-Line Therapies in Patients With PD-L1 TPS $\geq 1 \%$ Metastatic NSCLC. Cancers (Basel) (2020) 12(12):3648. doi: 10.3390/cancers 12123648

22. Salas-Benito D, Pérez-Gracia JL, Ponz-Sarvisé M, Rodriguez-Ruiz ME, Martínez-Forero I, Castañón E, et al. Paradigms on Immunotherapy Combinations With Chemotherapy. Cancer Discovery (2021). doi: 10.1158/ 2159-8290

23. Cavazzoni A, Digiacomo G, Alfieri R, La Monica S, Fumarola C, Galetti M, et al. Pemetrexed Enhances Membrane PD-L1 Expression and Potentiates T CellMediated Cytotoxicity by Anti-PD-L1 Antibody Therapy in Non-Small-Cell Lung Cancer. Cancers (Basel) (2020) 12(3):666. doi: 10.3390/cancers12030666

24. Sharma P, Allison JP. Dissecting the Mechanisms of Immune Checkpoint Therapy. Nat Rev Immunol (2020) 20(2):75-6. doi: 10.1038/s41577-020-0275-8

25. Wei SC, Duffy CR, Allison JP. Fundamental Mechanisms of Immune Checkpoint Blockade Therapy. Cancer Discovery (2018) 8(9):1069-86. doi: 10.1158/2159-8290.CD-18-0367

26. Planchard D, Popat S, Kerr K, Novello S, Smit EF, Faivre-Finn C, et al. Correction to: "Metastatic non-Small Cell Lung Cancer: ESMO Clinical Practice Guidelines for Diagnosis, Treatment and Follow-Up". Ann Oncol (2019) 30(5):863-70. doi: 10.1093/annonc/mdy474

27. Boyer M, Şendur MAN, Rodríguez-Abreu D, Park K, Lee DH, Çiçin I, et al. Pembrolizumab Plus Ipilimumab or Placebo for Metastatic Non-Small-Cell 
Lung Cancer With PD-L1 Tumor Proportion Score $\geq 50 \%$ : Randomized, Double-Blind Phase III KEYNOTE-598 Study. J Clin Oncol (2021). doi: 10.1200/JCO.20.03579. JCO2003579.

28. Shigematsu Y, Hanagiri T, Shiota H, Kuroda K, Baba T, Mizukami M, et al. Clinical Significance of Cancer/Testis Antigens Expression in Patients With non-Small Cell Lung Cancer. Lung Cancer (2010) 68(1):105-10. doi: 10.1016/ j.lungcan.2009.05.010

29. Gure AO, Chua R, Williamson B, Gonen M, Ferrera CA, Gnjatic S, et al. CancerTestis Genes are Coordinately Expressed and are Markers of Poor Outcome in Non-Small Cell Lung Cancer. Clin Cancer Res (2005) 11(22):8055-62. doi: 10.1158/1078-0432.CCR-05-1203

30. Klein SL, Flanagan KL. Sex Differences in Immune Responses. Nat Rev Immunol (2016) 16(10):626-38. doi: 10.1038/nri.2016.90

31. Franconi F, Brunelleschi S, Steardo L, Cuomo V. Gender Differences in Drug Responses. Pharmacol Res (2007) 55(2):81-95. doi: 10.1016/j.phrs. 2006.11.001

32. Kim AM, Tingen CM, Woodruff TK. Sex Bias in Trials and Treatment Must End. Nature (2010) 465(7299):688-9. doi: 10.1038/465688a

33. Pope CA 3rd, Burnett RT, Turner MC, Cohen A, Krewski D, Jerrett M, et al. Lung Cancer and Cardiovascular Disease Mortality Associated With Ambient Air Pollution and Cigarette Smoke: Shape of the Exposure-Response Relationships. Environ Health Perspect (2011) 119(11):1616-21. doi: 10.1289/ ehp.1103639

34. Li Y, Wang Z, Nair A, Song W, Yang P, Zhang X, et al. Comprehensive Profiling of lincRNAs in Lung Adenocarcinoma of Never Smokers Reveals Their Roles in Cancer Development and Prognosis. Genes (Basel) (2017) 8 (11):321. doi: 10.3390/genes8110321

35. Condoluci A, Mazzara C, Zoccoli A, Pezzuto A, Tonini G. Impact of Smoking on Lung Cancer Treatment Effectiveness: A Review. Future Oncol (2016) 12 (18):2149-61. doi: 10.2217/fon-2015-0055

36. Givi ME, Folkerts G, Wagenaar GT, Redegeld FA, Mortaz E. Cigarette Smoke Differentially Modulates Dendritic Cell Maturation and Function in Time. Respir Res (2015) 16:131. doi: 10.1186/s12931-015-0291-6
37. Herberth G, Bauer M, Gasch M, Hinz D, Röder S, Olek S, et al. Maternal and Cord Blood miR-223 Expression Associates With Prenatal Tobacco Smoke Exposure and Low Regulatory T-Cell Numbers. J Allergy Clin Immunol (2014) 133(2):543-50. doi: 10.1016/j.jaci.2013.06.036

38. Kerdidani D, Magkouta S, Chouvardas P, Karavana V, Glynos K, Roumelioti F, et al. Cigarette Smoke-Induced Emphysema Exhausts Early Cytotoxic CD8+ T Cell Responses Against Nascent Lung Cancer Cells. J Immunol (2018) 201 (5):1558-69. doi: 10.4049/jimmunol.1700700

39. Larkin J, Hodi FS, Wolchok JD. Combined Nivolumab and Ipilimumab or Monotherapy in Untreated Melanoma. N Engl J Med (2015) 373(13):1270-1. doi: 10.1056/NEJMc1509660

40. Wei SC, Anang NAS, Sharma R, Andrews MC, Reuben A, Levine JH, et al. Combination Anti-CTLA-4 Plus Anti-PD-1 Checkpoint Blockade Utilizes Cellular Mechanisms Partially Distinct From Monotherapies. Proc Natl Acad Sci USA (2019) 116(45):22699-709. doi: 10.1073/pnas.1821218116

Conflict of Interest: The authors declare that the research was conducted in the absence of any commercial or financial relationships that could be construed as a potential conflict of interest.

Publisher's Note: All claims expressed in this article are solely those of the authors and do not necessarily represent those of their affiliated organizations, or those of the publisher, the editors and the reviewers. Any product that may be evaluated in this article, or claim that may be made by its manufacturer, is not guaranteed or endorsed by the publisher.

Copyright (C) 2021 Jiang, Mao, Wang, Jia, Geng, Xu, Jiang, Yang, Jiao and Guo. This is an open-access article distributed under the terms of the Creative Commons Attribution License (CC BY). The use, distribution or reproduction in other forums is permitted, provided the original author(s) and the copyright owner(s) are credited and that the original publication in this journal is cited, in accordance with accepted academic practice. No use, distribution or reproduction is permitted which does not comply with these terms. 